\title{
Effects of molecular potential and geometry on atomic core-level photoemission over an extended energy range: The case study of the $\mathrm{CO}$ molecule
}

\author{
E. Kukk,${ }^{1, *}$ D. Ayuso, ${ }^{2}$ T. D. Thomas, ${ }^{3}$ P. Decleva,${ }^{4, \dagger}$ M. Patanen,${ }^{5}$ L. Argenti, ${ }^{2}$ E. Plésiat,${ }^{2}$ A. Palacios, ${ }^{2}$ K. Kooser, ${ }^{1}$ \\ O. Travnikova, ${ }^{5}$ S. Mondal, ${ }^{6}$ M. Kimura, ${ }^{6}$ K. Sakai, ${ }^{6}$ C. Miron, ${ }^{5}$ F. Martín,,${ }^{2,7}$ and K. Ueda ${ }^{6, \ddagger}$ \\ ${ }^{1}$ Department of Physics and Astronomy, University of Turku, FIN-20014 Turku, Finland \\ ${ }^{2}$ Departamento de Química, Módulo 13, Universidad Autónoma de Madrid, Cantoblanco 28049 Madrid, Spain \\ ${ }^{3}$ Department of Chemistry, Oregon State University, Corvallis, Oregon 97331, USA \\ ${ }^{4}$ Dipartimento di Scienze Chimiche, Università di Trieste, and CNR-IOM, 34127 Trieste, Italy \\ ${ }^{5}$ Synchrotron SOLEIL, L'Orme des Merisiers, Saint-Aubin, B.P. 48, 91192 Gif-sur-Yvette Cedex, France \\ ${ }^{6}$ Institute of Multidisciplinary Research for Advanced Materials, Tohoku University, Sendai 980-8577, Japan \\ ${ }^{7}$ Instituto Madrileño de Estudios Avanzados en Nanociencia, Cantoblanco, 28049 Madrid, Spain
}

(Received 26 April 2013; published 10 September 2013)

\begin{abstract}
We report an experimental and theoretical study of single-molecule inner-shell photoemission measured over an extended range of photon energies. The vibrational intensity ratios $I(v=1) / I(v=0)$ from the $\mathrm{C} 1 s$ photoelectron spectra of carbon monoxide, although mostly determined by the bond length change upon ionization, are shown to be affected also by photoelectron recoil and by scattering from the neighboring oxygen atom. Static-exchange density functional theory (DFT) is used to encompass all these effects in a unified theoretical treatment. The $a b$ initio calculations show that the vibrational ratio as a function of the photoelectron momentum is sensitive to both the ground-state internuclear distance and its contraction upon photoionization. We present a proof-of-principle application of DFT calculations as a quantitative structural analysis tool for extracting the dynamic and static molecular geometry parameters simultaneously.
\end{abstract}

DOI: 10.1103/PhysRevA.88.033412

PACS number(s): 33.80.Eh, 32.80.Aa, 33.15.-e, 33.20.-t

\section{INTRODUCTION}

Diatomic molecules in the gas phase have long been of fundamental scientific interest as the simplest quantum mechanical systems combining fast (electronic) and slow (nuclear) dynamics. Electronic structure studies of molecules ranging from the diatomics to complex organic molecules rely largely on energy- or wavelength-resolving spectroscopies. While the parameters of nuclear geometry of small molecules can be successfully derived using spectroscopic techniques, larger systems are probed mainly with scattering techniques in which either an electromagnetic or a matter wave is perturbed by atoms, creating new emission centers of scattered waves. Continuum waves from various sources then overlap and interfere, creating a characteristic pattern of intensity modulations. The suitability of scattering techniques for molecular structure determination in the gas phase was established in 1915 by Debye, who showed that, contrary to what might be assumed, averaging over the random orientations of free molecules containing the scattering atoms does not destroy the interference patterns [1]. Instead, pronounced oscillations of the outgoing wave intensity as a function of the detection angle are expected, with a period that depends on the structural parameters of the molecule such as its bond lengths, and on the wavelength of the scattered wave.

The scattering techniques can employ a variety of particles to form the wave to be scattered: electrons, photons, and neutrons. Due to the strong interaction of charged particles

\footnotetext{
*edwin.kukk@utu.fi

†decleva@units.it

${ }^{\ddagger}$ ueda@tagen.tohoku.ac.jp
}

with matter, electron scattering has become a standard technique in the gas phase, where it can determine structural parameters of free molecules with high accuracy [2]. X-ray and neutron diffraction, on the other hand, are the methods of choice for solid targets. There is also a choice between the origin of the wave to be scattered: external, as in the methods mentioned above, or internal. In the latter, electrons emitted by one of the atoms in the system form the initial wave that is subsequently scattered by neighboring atoms. In this case, the photoemission cross section $\sigma(k)$ is modified by interference terms that oscillate as a function of the electron momentum $k$ as $\sim \sin \left(2 k R_{0}\right)$, where $R_{0}$ is the distance between the emitter and the scatterer. This effect is at the basis of EXAFS (extended x-ray absorption fine structure) [3], a technique broadly employed in solids, and in which the short-range neighborhood structure is revealed by oscillations in the x-ray absorbance. In the less broadly employed technique of XPD (x-ray photoelectron diffraction), structural information of surfaces is extracted directly from photoemission intensity distributions, either angle or momentum resolved [4,5].

In randomly oriented molecular targets, in contrast to solids, photoemission has not been the tool of choice to probe the static geometry of the emitter's neighborhood. Instead, core-level photoelectron spectroscopy was found to be an excellent means to elicit information on molecular dynamics: the disturbance to the electronic structure by core hole creation induces changes in equilibrium structural parameters, causing readjustment of atomic positions and resulting in vibrational excitations [6]. In the case of diatomics, for example, the change of the bond length $\Delta R$ is derived from the analysis of the vibrational progression in the photoelectron spectrum via Franck-Condon factors $[7,8]$. 
The derivation of static $\left(R_{0}\right)$ and dynamic $(\Delta R)$ structural parameters has thus been largely based on different and independent approaches. A number of recent studies of molecular photoemission over extended energy ranges (e.g., [9-11]), however, made apparent that these properties should not be treated separately in the first place. Firstly, the emission of a photoelectron with momentum $k$ entails a corresponding nuclear recoil that induces vibrational and rotational excitations. Yet, the direction of the nuclear recoil depends on whether the electron leaves the molecule directly or scatters on neighboring atoms. Secondly, scattering and interference effects modulate the cross sections and also affect the ratios of the vibrational peaks in the spectrum [11]. Thus, a highly accurate determination of $\Delta R$ from a photoelectron spectrum at any given energy requires that these effects, which depend on the static molecular geometry, be taken into account as well.

In this study, we analyze the extended range photoemission spectrum of carbon monoxide (CO), a simple diatomic system, using an advanced theoretical model that includes all of the aforementioned effects. The focus of the study is on the ratio of vibrational intensities in the $\mathrm{C} 1 \mathrm{~s}$ photoemission, $(v=1) /(v=0)$, as obtained from the experiment and by $a b$ initio methods. We will refer to these as the "v-ratios." The v-ratios are primarily determined by the change $(\Delta R)$ of the internuclear equilibrium distance and are calculated by means of the Franck-Condon factors. In the present case, both the recoil and intramolecular photoelectron scattering modify the Franck-Condon v-ratios. This circumstance is to be contrasted with the case of core-photoionization of the central atom in symmetric $A B_{n}$ molecules $\left(\mathrm{BF}_{3}\right.$ [12], $\mathrm{CF}_{4}$ [10,12,13], and $\left.\mathrm{CH}_{4}[9,11]\right)$, where the photoelectron is ejected from the center of mass (hence, there are no rotational excitations) and the recoil and Franck-Condon excitations affect different vibrational modes $[9,10]$. As a result, the analysis of a diatomic molecule as simple as $\mathrm{CO}$ is in fact more complex than that of some larger systems with many vibrational degrees of freedom.

We have employed static-exchange density functional theory to obtain ab initio vibrationally resolved cross-sections of the C $1 s$ photoemission for $v=0$ and $v=1$ levels. These molecular effects are superimposed on the atomic cross section, which falls off sharply with increasing photon energy. As a result, direct comparison of theoretical cross sections with experiment is a difficult task with uncertain outcome. For this reason we compare the $\mathrm{v}$-ratios, which are relative values, instead. We show in a systematic approach how the bond contraction, intramolecular scattering ( $R_{0}$-dependent effect), and recoil excitations together determine the v-ratios observed in the $\mathrm{C} 1 s$ photoelectron spectra. This work is also a proof of principle that such a theoretical approach can be used to determine both the static and dynamic structural properties of a molecule at the same time.

\section{C $1 s$ PHOTOELECTRON SPECTRA: EXPERIMENTS AND DATA TREATMENT}

The experimental values of the intensity ratios in the vibrational progression in $\mathrm{C} 1 s$ photoelectron spectra of gasphase carbon monoxide molecules were extracted from spectra measured over several different experimental periods and at

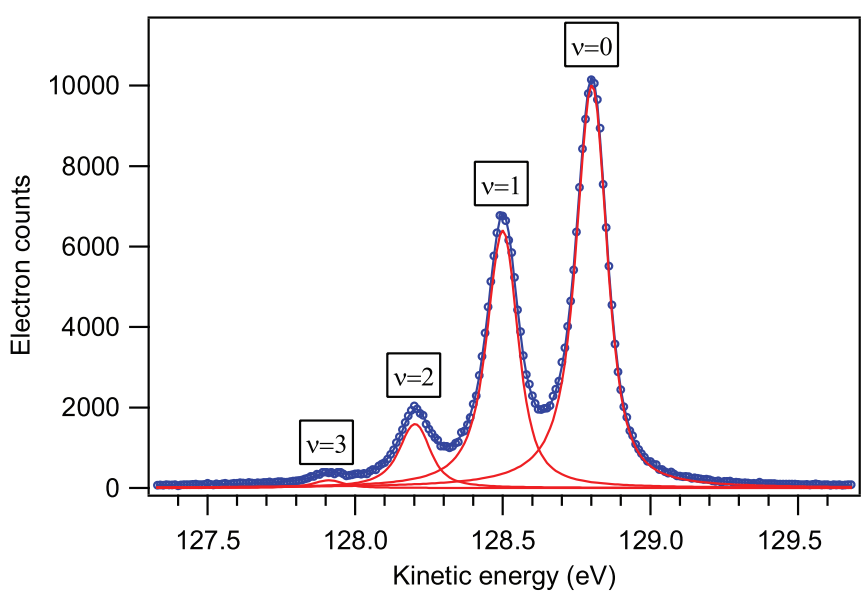

FIG. 1. (Color online) Carbon $1 s$ photoelectron spectrum, measured at $h v=425 \mathrm{eV}$ at SOLEIL. Cirles: experiment; solid lines: least-squares fit of the vibrational progression.

two synchrotron radiation facilities, SPring-8 (Japan) and SOLEIL (France). The details for the experimental setups used at beamline BL27SU of SPring-8 and at beamline PLEIADES of SOLEIL are described in Refs. [14] and [15], respectively. In brief, Scienta 200-mm mean radius hemispherical electron energy analyzers were used in both experiments, while linearly polarized undulator radiation was used and the target gas was contained in a differentially pumped gas cell. The photon energy range covered by the PLEIADES dataset is from 320 to $525 \mathrm{eV}$ and the electrons were detected at the "magic" emission angle of $57.4^{\circ}$ from the polarization vector of linearly polarized light. The SPring- 8 datasets from several experimental periods cover a wider photon energy range from 350 to $1500 \mathrm{eV}$. These spectra were recorded at $0^{\circ}$ from the linear polarization vector. In order to compare the intensity ratios with the PLEIADES spectra taken at the magic angle, the following conversion should be applied:

$$
R_{54.7}=\frac{R_{0}+2 c R_{90}}{1+2 c}, \quad c=\frac{1-\beta / 2}{1+\beta} .
$$

Here, $\beta$ is the anisotropy parameter of $\mathrm{C} 1 s$ photoemission. At the photon energy of $330 \mathrm{eV}$, both the experimental $(\beta=1.79(5)[16])$ and theoretical values $(\beta=1.7$ [17]) are already close to the maximum value of 2 . As $\beta$ asymptotically approaches the maximum value at higher energies, $c \rightarrow 0$. In the higher energy range of the present study, in a good approximation $R_{54.7} \approx R_{0}$. Therefore, in this work we compare the v-ratios obtained from $0^{\circ}$ and magic-angle spectra directly. Figure 1 shows one example of the spectra analyzed for vratios, measured at the PLEIADES beamline. The instrumental broadening of the peaks arises as convolution of the spectrometer's resolution (about $38 \mathrm{meV}$ FWHM) and photon bandwidth (about $41 \mathrm{meV}$ FWHM). Other experimental contributions to the line width in the spectra come from the translational and rotational [18] Doppler broadenings, which in the case of the spectrum shown in Fig. 1 are 28 and $25 \mathrm{meV}$, respectively. The spectra were decomposed into individual vibrational components by least-squares fitting using the SPANCF macro package for Igor Pro [19]. The experimental broadenings were represented by Gaussian profiles of adjustable width. 
In the PLEIADES spectra that width ranged from 42 to 88 meV FWHM; in the SPring-8 spectra from 61 to $250 \mathrm{meV}$ FWHM depending on the photon energy. These Gaussian profiles were convoluted with distorted Lorentzian shapes arising from the natural width of the $\mathrm{C} 1 s^{-1}$ core hole state. The asymmetric distortion is caused by post-collision interaction (PCI) between the photoelectron and the Auger electron and it was represented by analytical PCI lineshapes of van der Straten [20]. The PCI asymmetry appears only at photon energies $h v \lesssim 550 \mathrm{eV}$. The lifetime width was represented by a common value for each dataset analyzed; all datasets except one from Spring-8 converged to values within $92.0 \pm 1.0 \mathrm{meV}$, in good agreement with the value of $95 \pm 5 \mathrm{meV}$ reported by Carroll et al. [8]. (The deviant value from one dataset was caused by a significant Lorentzian component in the instrumental broadening.) Energies (for $v=0$ peaks) and spacings $\Delta E_{0, v}$ (from $v>0$ peaks to $v=0$ peak) were also given as adjustable fit parameters, but linked to be the same for each spectum in a dataset. The spacings from the highest quality PLEIADES dataset are $\Delta E_{0,1}=302, \Delta E_{1,2}=298$, and $\Delta E_{2,3}=288 \mathrm{meV}$.

\section{THEORY}

Vibrationally resolved cross sections are evaluated with first-order perturbation theory within the Born-Oppenheimer (BO) and the dipole approximations:

$$
\sigma_{\alpha}\left(v, v^{\prime}, \omega\right)=\frac{4 \pi^{2} \omega}{3 \hbar c} a_{0}^{2} \sum_{\eta} \sum_{l_{\eta}}\left|T_{\alpha_{\eta} l_{\eta} v v^{\prime}}(\varepsilon)\right|^{2},
$$

where $v$ and $v^{\prime}$ are the vibrational quantum numbers for the neutral molecule and for the cation, respectively $(v=0$ since the initial state is the ground state of $\mathrm{CO}$ ), $\omega$ is the photon energy, $\varepsilon$ is the photoelectron kinetic energy, $\alpha$ denotes the electronic state of the residual cation, $l$ is the angular momentum quantum number, and $\eta$ stands for the symmetry of the final state. The BO approximation permits to write the transition element $T_{\alpha_{\eta} l_{\eta} v v^{\prime}}(\varepsilon)$ as

$$
T_{\alpha_{\eta} l_{\eta} v v^{\prime}}(\varepsilon)=\int \chi_{\mathrm{CO}, v}^{*}(R) \mu_{\alpha_{\eta} l_{\eta}}(R) \chi_{\mathrm{CO}_{\alpha}^{+}, v^{\prime}}(R) d R,
$$

where $\chi_{\mathrm{CO}_{\alpha}, v}$ and $\chi_{\mathrm{CO}_{\alpha}^{+}, v^{\prime}}$ are the initial and the final vibrational wave functions and $\mu_{\alpha_{\eta} l_{\eta}}$ is the dipole coupling element between the initial electronic state, $\phi_{0}$, and the final one, $\phi_{\alpha_{\eta} l_{\alpha}}$, which in this case is obtained by promoting one electron from the $2 \sigma$ molecular orbital of $\mathrm{CO}$ to a continuum orbital. Electronic wave functions have been evaluated in a grid of 56 internuclear distances around the equilibrium geometry, as in previous work [21], employing the staticexchange density functional theory (DFT) method, developed by Decleva and co-workers [22,23]. This method makes use of the Kohn-Sham DFT to describe the molecular ionic states and the Galerkin approach to evaluate continuum electron wave functions in the field of the corresponding Kohn-Sham density. A standard local density approximation functional has been used to describe electronic exchange and correlation effects. Vibrational eigenfunctions have been obtained by solving the time-independent Schrödinger equation in a basis set of 256 B-splines within a box of 10 a.u. We have employed the Morse approximation to construct potential energy curves from reliable spectroscopic parameters available in the literature [24]. The calculated v-ratios depend primarily on the equilibrium bond lengths in the neutral molecule and the cation, and especially on the difference in bond lengths, $\Delta R$. For instance, the v-ratios are modified approximately by $20 \%$ for a variation of $1 \%$ in $\Delta R$, whilst the effect of similar changes in $\omega_{e}$ or in $\omega_{e} x_{e}$ is only between $0.1 \%$ and $1 \%$. This behavior is due to (1) the Franck-Condon overlap, which, without affecting the total cross section, changes dramatically the contribution from each vibrational excitation; and (2) the $R$ dependence of the dipole elements [see Eq. (3)].

\section{ANALYSIS}

\section{A. Static-exchange DFT results}

Before comparing the theoretical results with experimental data points, let us first consider the features of the theoretical curves in some detail. Figure 2 shows a set of static-exchange DFT calculations for the v-ratios in $\mathrm{C} 1 s$ photoemission spectra as a function of electron momentum, for several values of two crucial parameters, the ground state internuclear distance $R_{0}$ and the contraction of that distance upon core ionization, $\Delta R$. The bold curves in both panels of Fig 2 are from a calculation based on the literature values $R_{\text {lit }}=$ 2.1322 a.u. [25] and $\Delta R_{\text {lit }}=-0.0932$ a.u. [7]. For comparison, the constant Franck-Condon v-ratio computed from the eigenfunctions of the Morse potentials is also reported. This value, $I(v=1) / I(v=0)=0.6110$, is obtained from $R_{0}=2.1322$ a.u., $\Delta R=-0.0932$ a.u., and the Morse curve parameters $\omega_{e}=269.0 \mathrm{meV}, \omega_{e} \chi_{e}=1.648 \mathrm{meV}$ and $\omega_{e}=$ $304.6 \mathrm{meV}, \omega_{e} \chi_{e}=1.24 \mathrm{meV}$ for the ground and core-ionized states, respectively [24]. The set of curves in panel (a) of Fig. 2 is obtained by varying the ground-state internuclear distance in the range of $R_{0}=(0.95-1.05) R_{\text {lit }}$. The effect of this variation is seen mainly as a change in the oscillation period, with shorter periods corresponding to larger internuclear distances. This is consistent with the picture where the oscillations are due to the interference between the electron emitted directly from the carbon atom and the same electron scattered by the oxygen atom. Indeed, a simplified analytical formulation of the interference pattern based on the interatomic scattering mechanism predicts that the period of the v-ratios' oscillations in the momentum scale is $\Delta k=\pi / R_{0}$ [11]. This is also the period that appears in the well known EXAFS equation [3], where each $\sin \left(2 R_{j} k+\delta\right)$ term represents photoelectron scattering from the $j$ th near-neighbor shell in crystalline solid.

Assuming an internuclear distance $R_{0}$ of 2.1322 a.u., the static-exchange DFT calculation predicts an oscillation period of about $\Delta k=1.9$ a.u. in momentum units (Fig. 2, bold curves). But according to the relationship $\Delta k=\pi / R_{0}$ mentioned above, this value should correspond to an internuclear distance $R_{0}=1.64$ a.u. that is considerably smaller than the value provided as input. This discrepancy, which indicates how simplified analytical expressions are inadequate for quantitative structure determination, may have several causes. Firstly, the expression leading to the oscillation period of $\pi / R_{0}$, as in the EXAFS equation, is based on the assumption that only single-scattering events are relevant. As a rule 

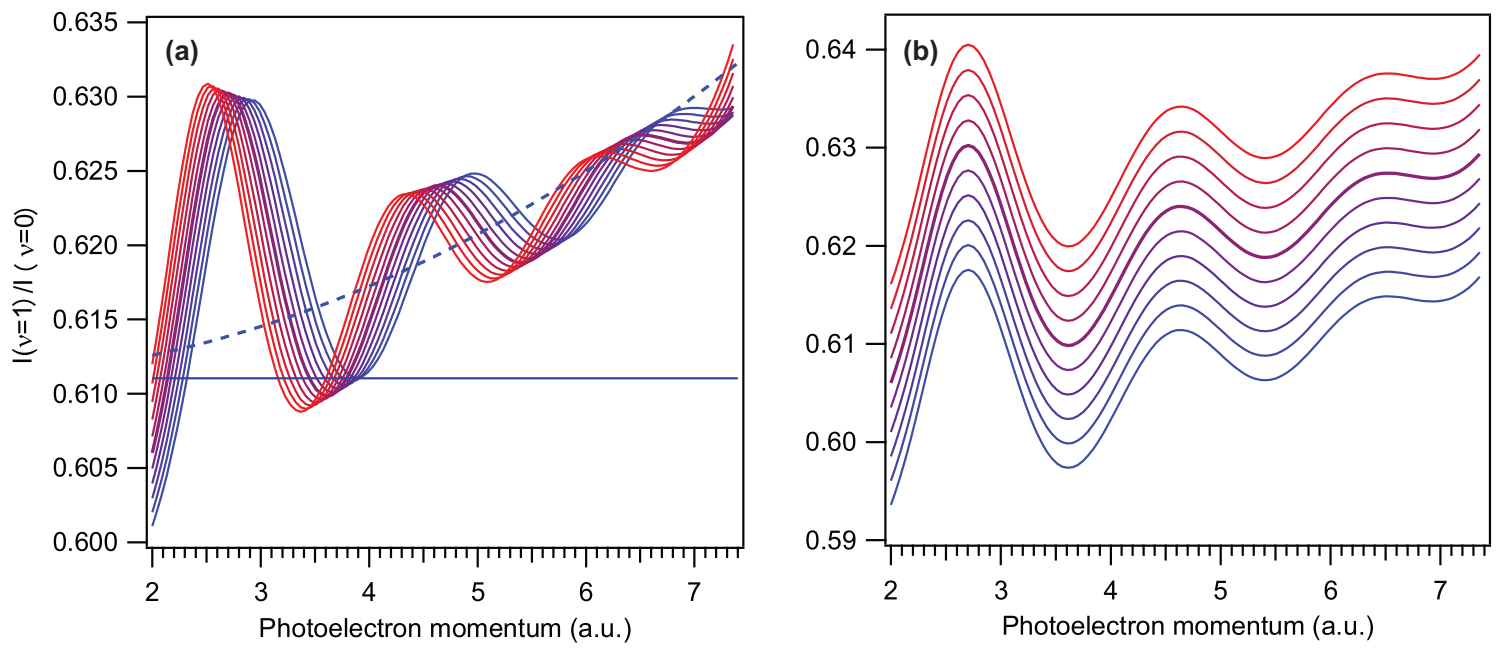

FIG. 2. (Color online) Static-exchange DFT calculations of vibrational ratios. Bold middle curves in both panels are obtained with $R_{0}=R_{\text {lit }}$ and $\Delta R=\Delta R_{\text {lit }}$. (a) Variations of $R_{0}=0.95 \ldots 1.05 R_{\text {lit }}$ (from blue to red) and (b) $\Delta R=0.99 \ldots 1.01 \Delta R_{\text {lit }}$ (from blue to red). Horizontal line in panel a marks the value from Franck-Condon calculation and the the dashed curve reprents the photoelectron recoil effect.

of thumb, in EXAFS analysis this is considered a good assumption provided that $k \gtrsim 1.5$ a.u. [3]. Since in this study we investigate the range of $k>2$ a.u., multiple scattering can be expected to have only minor effects. Secondly, as it is known also for the EXAFS equation, the phase $\delta$ of the oscillation (or, equivalently, the oscillation frequency) is $k$ dependent, while in the simplified analytical treatment it is considered constant.

One can see that, apart from the scattering-induced oscillations, the DFT prediction also systematically deviates from the Franck-Condon constant value as the photoelectron energy increases. This deviation is due to the photoelectron recoil effect, represented in Fig. 2(a) by a dashed curve, whose contribution to the v-ratio is estimated as follows: The Franck-Condon v-ratio provides the baseline for the recoil contribution. In the momentum scale, the photoelectron recoil excitation probability from $v=0$ to the $v=1$ level increases quadratically, and so does the corresponding v-ratio. The dashed line in Fig. 2(a) shows the photoelectron recoil contribution computed as

$$
F(k)=F_{\mathrm{FC}}+c_{\text {rec }} k^{2},
$$

where $F_{\mathrm{FC}}$ is the Franck-Condon baseline and the the recoil coefficient $c_{\text {rec }}$ is calculated following the semiclassical model given in Refs. [9,10]. The recoil coefficient also accounts for the recoil momentum partitioning between vibrational and rotational excitations and depends on the photoelectron angular distribution in the molecular frame (MFPAD). We made an approximation that, after averaging over the angles between the linear polarization vector of the x-rays and the random laboratory frame orientations of the molecular axis, MFPAD becomes isotropic and then

$$
c_{\text {rec }}=3.87 \times 10^{-4} \text { a.u. }^{-2} .
$$

On one hand, recent results using the COLTRIMS (cold target recoil ion momentum spectroscopy) technique have shown that at low photoelectron kinetic energies the MFPADs, even after averaging over the direction of the polarization vector, can be strongly anisotropic (e.g., photoemission preferentially along the molecular bonds in methane) [26]. Indications of molecular frame anisotropy at low energies are given also by are given also by the occurrence of "knockout" secondary ionization processes induced by the primary photoelectron [27]. On the other hand, it was also observed that MFPAD rapidly approaches isotropic distribution with increasing electron energy [26] and we thus believe that in the range of strong recoil effects the approximation of isotropic orientationally averaged MFPAD is well justified, although the recoil model would benefit from more detailed information on MFPADs at the energies of interest.

As can be seen from Fig. 2(a), the recoil model reproduces well the average rising trend of the DFT calculation. Indeed, the calculation is expected to reproduce the recoil, provided that the coordinate system is set at the center of mass of the molecule (as was the case here). In Eq. (4), the Franck-Condon and recoil excitations probabilities are added incoherently. Using the generalized Franck-Condon factors [28,29], it can be shown that this approach is appropriate for the core-ionization from heteronuclear diatomics, where the core orbitals are well localized to a single atom. However, there is also the possibility that scattering of the outgoing photoelectron will cause the molecular frame angular distribution of the photoelectron to be other than isotropic. In this case, the coefficient $c_{\text {rec }}$ in Eq. (4) would differ from the constant value indicated above and could depend on the kinetic energy as well. All these effects are included in the static-exchange DFT calculations; the near agreement between the trend of these calculations and the predictions of Eq. (4) confirms that, in the present case, these effects are small.

Panel (b) of Fig. 2 illustrates the changes in the v-ratios at fixed internuclear distance $R_{0}=R_{\text {lit }}$, varying the bond contraction $\Delta R$ upon core ionization in the range $\Delta R=$ $(0.99-1.01) \Delta R_{\text {lit }}$. As seen from the figure, even a tiny variation of $\Delta R$ results in large vertical excursion of the v-ratios' curves. These variations would be reflected also in the baseline Franck-Condon factors as the displacement along $R$ of the initial and final state potential energy curves changes. Figure 2 
thus demostrates that the molecular v-ratios are sensitive probes for both the static and dynamic structure parameters. In the next section, these calculations are compared with the experimental results.

\section{B. Comparison with experiment and derivation of geometrical parameters}

The ratios of the intensities of the $v=1$ and $v=0$ vibrational peaks in $\mathrm{C} 1 s$ experimental photoelectron spectra, extracted by least-squares curve fitting, are shown in Fig. 3. The error bars of the SOLEIL data represent the statistical standard deviations obtained from the fitting. In the SPring- 8 datasets it was observed that, when analyzing repeated electron energy scans at a particular energy, the v-ratios varied more than the error estimates based on counting statistics, most likley due to instabilities in electron detection. The error bars for this latter set take into account also this additional source of variation. In addition to the results from these experiments, the three data points with the lowest $k$ values were extracted from the spectra published by Carroll et al. [8]. More values for the v-ratios in $\mathrm{C} 1 s$ photoionization of $\mathrm{CO}$ are available in literature [30,31], but these were not included for comparison, because we did not have the possibility to reanalyze the original spectra consistently with the same methods.

The thin red line once again shows the DFT calculation based on the literature values $R_{\text {lit }}=2.1322$ a.u. [25] and $\Delta R_{\text {lit }}=-0.0932$ a.u. [8]. Below $k=2$ a.u. the theory is in excellent agreement with the experimental data, representing also the major increase of the v-ratio due to the presence of the shape resonance near the threshold $[11,32]$. However, at $k \geqslant 2$ a systematic discrepancy appears. This vertical offset of the theory is mostly influenced by the choice of the value of $\Delta R$. In considering this discrepancy, one should keep in mind that the literature value for $\Delta R_{\text {lit }}$ has in fact been

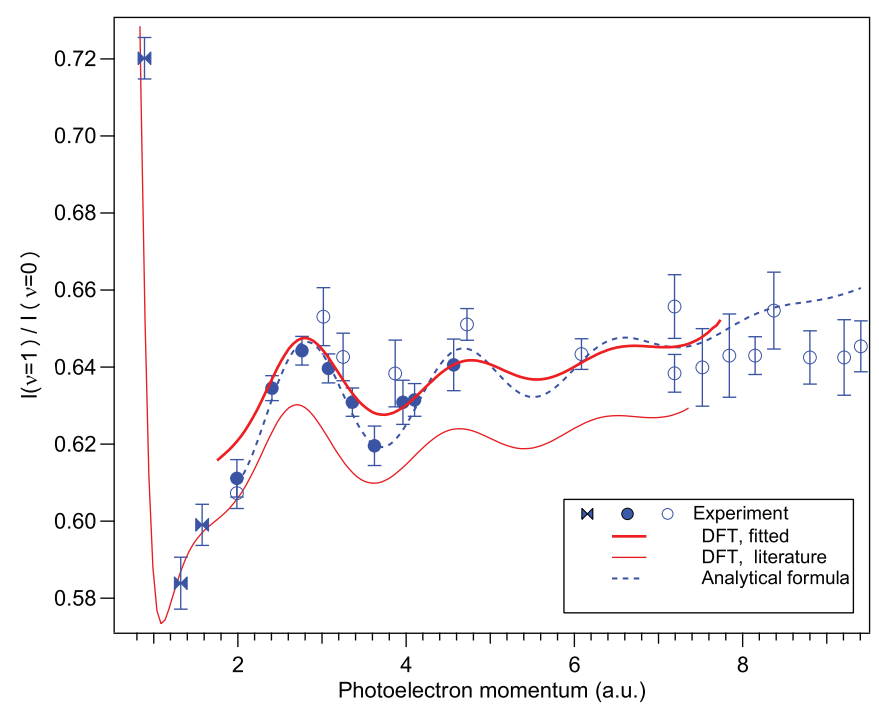

FIG. 3. (Color online) Experimental and calculated intensity ratios of the vibrational peaks in $\mathrm{C} 1 s$ photoelectron spectra of $\mathrm{CO}$. Open cirles: measurements at SPring8; full circles: measuments at SOLEIL; double triangles: Ref. [8]. Bold solid red line: fit of the static-exchange DFT theory; thin red line: DFT theory with literature input values; dashed blue line: fit of analytical formula. obtained from basic Franck-Condon analyis of photoelectron spectra such as the one depicted in Fig. 1. Yet Fig. 2 clearly shows that such an analysis does not properly take into account the modulation due to intramolecular scattering and photoelectron recoil, thus resulting in uncertain estimates of the internuclear bond contraction. Furthermore, the shape resonance in the $\mathrm{C} 1 s$ photoionization of $\mathrm{CO}$ [32] affects the cross sections for the electrons with momenta up to $\approx 1-2$ a.u. above threshold [11]. Since shape-resonance maxima occur at slightly different energies for different vibrationally resolved cross sections (by about $2 \mathrm{eV}$ ), the resonance also distorts the v-ratios close to threshold, rendering them unsuitable for accurate Franck-Condon analysis. In the energy range above the shape resonance, it is the scattering oscillations that modify the v-ratios. Finally, at high energy, where the oscillations are strongly damped, recoil excitations start to contribute significantly. It is therefore not surprising that calculations based on available literature data may fail to represent the experimenal v-ratios across a broad electron momentum range.

In order to perform a consistent derivation of geometrical parameters from the experimental v-ratios, a least-squares fit of the DFT calculation to the experiment was performed. The result is shown in Fig. 3 as a bold red curve (also indicating the range of data points that were included in the fit). As the span over $k$ of the fitted curve indicates, the data points for the lowest and highest momenta were not included since the purpose was the derivation of the geometrical parameters. The near-threshold values of the v-ratios are strongly influenced by multiple scattering and the shape resonance, while at large momentum values the characteristic oscillations become significantly damped. Also the near-threshold region is more sensitive to electron correlation and less accurately described by the static DFT calculations.

The fitting variables were $R_{0}$ and $\Delta R$. The best agreement with the experimental data (as measured by $\chi^{2}$ goodnessof-fit value) was obtained with $R_{0}=2.09 \pm 0.05$ a.u. and $\Delta R=-0.0945 \pm 0.0002$ a.u. The uncertainties are estimated from the fitting procedure and depend on the number of the experimental data points and their error bars; the uncertainties do not reflect any inaccuracies in the theoretical model. Such a quantitative procedure is feasible because, although the integral in Eq. (3) must be reevaluated at each step of the optimization procedure (the vibrational wave functions depend on the values of the Morse parameters), the dipole matrix elements $\mu_{\alpha_{\eta} l_{\eta}}(R)$ are computed once and for all at once. As a consequence, the fitting algorithm is actually very efficient from the computational point of view.

To get a better insight into the different contributions to the v-ratios, we added the oscillatory term of the simplified analytical model to the photoelectron recoil curve [Eq. (4)]:

$$
F=F_{\mathrm{FC}}+A \exp \left(-c k^{2}\right) \sin (2 R k+\delta)+c_{\text {rec }} k^{2} .
$$

Once it was ascertained that Eq. (5) can match the fully theoretical curve with sufficient accuracy, the experimental data in Fig. 3 was fitted by freely varying the five parameters $F_{\mathrm{FC}}, A, c, R$, and $\delta$, while $c_{\text {rec }}$ was kept at the fixed value of $3.87 \times 10^{-4}$ a.u. ${ }^{-2}$, as explained above. As can be seen, the analytical formula follows the data well at $k$ less than 7 . However, it is notable that 7 out of 9 measured v-ratios at photon energies $\geqslant 1000 \mathrm{eV}(k \gtrsim 7)$ fall below the predicted 
recoil curve. Based on the available data, we cannot tell whether this discrepancy is due to a deficiency of the recoil model or is an experimental artifact. For example, in the fitting we assumed a Gaussian instrumental profile. If, however, the true instrument function is skewed towards high kinetic energy, the v-ratios extracted using a symmetric profile are too low. A fitting conducted with asymmetric profiles would bring the high-energy results into better agreement with the theoretical predictions, but at this point any quantitative assumption about peak asymmetry would be unjustified.

This least-squares fit to the experiment converged with the essential geometry-related parameters $F_{\mathrm{FC}}=0.627 \pm 0.001$ and $R=1.70 \pm 0.07$ a.u. As noted above, the analytical formula with EXAFS-like relationship between $R$ and the oscillation period provides a value significantly lower than the true bond length, while the DFT fit result of $R_{0}=2.09 \pm 0.06$ agrees well with the literature value of $R_{\text {lit }}=2.1322$ a.u. [25].

The literature values of $\Delta R_{\text {lit }}=-0.0932$ a.u. [24] and $-0.0917 \pm 0.0009$ a.u. from another source [8] are to be compared with the value of $\Delta R=-0.0945 \pm 0.0002$ a.u. obtained by fitting the static-exchange DFT theory to the experiment. The less accurate empirical formula [Eq. (5)] gave a Franck-Condon ratio that corresponds to $\Delta R=-0.096$ a.u.

We can see that employing the DFT theory allows for higly accurate determination of the dynamical parameter $\Delta R$, whereas the static ground state internuclear distance $R_{0}$ is determined much less accurately, particularly in comparison with other available methods such as microwave spectroscopy [25]. It is a unique feature of this method, however, that these two quantities are obtained simultaneously. Also, current errors originate mostly from the uncertainty in the experimental data and therefore future improvements in experimental statistics and resolution at higher energies would significantly improve the accuracy of the fit.

\section{CONCLUSION}

Vibrational intensity ratios (v-ratios) in the $\mathrm{C} 1 s$ photoelectron spectra of $\mathrm{CO}$ were investigated by comparing a combined experimental dataset from several experiments with $a b$ initio static-exchange DFT calculations, as well as with a simplified analytical model formula. The theoretical results included conventional Franck-Condon excitations, photoelectron recoil, and intramolecular scattering on the neighboring oxygen atom. It was demonstrated by a set of $a b$ initio curves that the v-ratios are potentially a very sensitive means of determining both static (internuclear distance) and dynamic (bond length change) geometrical parameters simultaneously, although improvements particularly in experimental values will be needed to realize this potential.
It was also noted that simplified theoretical models for analytical expressions of v-ratios, although providing an excellent means for qualitative interpretation, can easily lead to erroneous quantitative results. For quantitative structural determinations from experimental data, comparison with fully $a b$ initio theoretical descriptions is to be strongly preferred.

When theory and experiment are compared, it becomes apparent that bond lengths taken from the literature lead to a poor agreement. A least-squares fit of the full DFT calculation, varying $R_{0}$ and $\Delta R$, considerably improves the agreement. From the fit, we extracted the new geometry values $R_{0}=2.09$ a.u. and $\Delta R=-0.0945$ a.u. and demonstrated the feasibility of using the DFT calculations for geometry determination in molecules. Even if equilibrium internuclear distances are often accurately known for many molecular systems already, the uniqueness of the approach described here lies in the ability to determine both static and dynamic geometrical values simultenaously. In fact, a separate determination of the dynamical values only - the bond length change - the usual means of Franck-Condon analysis of the vibrational structure is inevitably prone to systematic errors, since the scattering effects are neglected. Regarding the practical use of this method for determining the dynamic structure parameters, we point out the local nature of the scattering, probing mostly the nearest neighbor distances (akin to EXAFS). Therefore, using well localized core ionization, one can probe particular regions of large molecules with accuracy potentially better than that achievable by other methods.

\section{ACKNOWLEDGMENTS}

We appreciate the help of the staff of SPring-8 (Japan) during the experiments there. At SOLEIL (France), data collection was performed at the PLEIADES beamline during beam time alloted under Proposal No. 20110781. SOLEIL staff are acknowledged for smoothly running the facilities and E. Robert for technical assistance. This work was supported by the FP7/2007-2013 Grant No. 252781 (OT) and Triangle de la Physique Contract No. 2007-010T (OT), and by the MEXT and JSPS agencies (Japan). We also thank Mare Nostrum BSC, Cineca and CCC-UAM for allocation of computer time. E.K. acknowledges financial support from the Academy of Finland. E.P., L.A., D.A., A.P., and F.M. acknowledge financial support from the Advanced Grant of the European Research Council XCHEM 290853, the MICINN Projects No. FIS2010-15127, No. ACI2008-0777, and No. CSD 2007-00010 (Spain), the ERA-Chemistry Project No. PIM2010EEC-00751, the European rants MC-ITN CORINF and MC-RG ATTOTREND, and the European COST Actions No. CM0702 (CUSPFEL) and No. CM1204 (XLIC).
[1] P. Debye, Ann. Phys. 46, 809 (1915).

[2] I. Hargittai, Electron Crystallogr. 211, 197 (2006).

[3] E. A. Stern, Phys. Rev. B 10, 3027 (1974).

[4] D. P. Woodruff, Appl. Phys. A 92, 439 (2008).

[5] F. Krasniqi, B. Najjari, L. Strüder, D. Rolles, A. Voitkiv, and J. Ullrich, Phys. Rev. A 81, 033411 (2010).
[6] Z. F. Liu, G. M. Bancroft, J. N. Cutler, D. G. Sutherland, K. H. Tan, J. S. Tse, and R. G. Cavell, Phys. Rev. A 46, 1688 (1992).

[7] B. Kempgens, K. Maier, A. Kivimäki, H. M. Köppe, M. Neeb, M. N. Piancastelli, U. Hergenhahn, and A. M. Bradshaw, J. Phys. B 30, L741 (1997). 
[8] T. X. Carroll, K. J. Børve, L. J. Sæthre, J. D. Bozek, E. Kukk, J. A. Hahne, and T. D. Thomas, J. Chem. Phys. 116, 10221 (2002).

[9] E. Kukk, K. Ueda, U. Hergenhahn, X.-J. Liu, G. Prümper, H. Yoshida, Y. Tamenori, C. Makochekanwa, T. Tanaka, M. Kitajima, and H. Tanaka, Phys. Rev. Lett. 95, 133001 (2005).

[10] T. D. Thomas, E. Kukk, R. Sankari, H. Fukuzawa, G. Prümper, K. Ueda, R. Püttner, J. Harries, Y. Tamenori, T. Tanaka, M. Hoshino, and H. Tanaka, J. Chem. Phys. 128, 144311 (2008).

[11] E. Plésiat, L. Argenti, E. Kukk, C. Miron, K. Ueda, P. Decleva, and F. Martín, Phys. Rev. A 85, 023409 (2012).

[12] K. Ueda et al., J. Chem. Phys (to be published).

[13] M. Patanen et al. (private communication).

[14] H. Ohashi, E. Ishiguro, Y. Tamenori, H. Kishimoto, M. Tanaka, M. Irie, T. Tanaka, and T. Ishikawa, Nucl. Instrum. Methods A 467, 529 (2001); K. Ueda, J. Phys. B 36, R1 (2003).

[15] O. Travnikova, J.-C. Liu, A. Lindblad, C. Nicolas, J. Söderström, V. Kimberg, F. Gel'mukhanov, and C. Miron, Phys. Rev. Lett. 105, 233001 (2010); J. Söderström, A. Lindblad, A. N. Grum-Grzhimailo, O. Travnikova, C. Nicolas, S. Svensson, and C. Miron, New J. Phys. 13, 073014 (2011); C. Miron, C. Nicolas, O. Travnikova, P. Morin, Y. Sun, F. Gel'mukhanov, N. Kosugi, and V. Kimberg, Nat. Phys. 8, 135 (2012).

[16] O. Hemmers, S. B. Whitfield, N. Berrah, B. Langer, R. Wehlitz, and U. Becker, J. Phys. B 28, L693 (1995).

[17] E. Plésiat, P. Decleva, and F. Martín, J. Phys. B 45, 194008 (2012).

[18] T. D. Thomas, E. Kukk, K. Ueda, T. Ouchi, K. Sakai, T. X. Carroll, C. Nicolas, O. Travnikova, and C. Miron, Phys. Rev. Lett. 106, 193009 (2011).
[19] E. Kukk, spectral analysis by curve fitting macro package, SPANCF 2000, http://www.physics.utu.fi/en/department/ materials_research/materials_science/Fitting.html.

[20] P. van der Straten, R. Morgenstern, and A. Niehaus, Z. Phys. D 8, 35 (1988).

[21] E. Plésiat, Phys. Chem. Chem. Phys. 14, 10853 (2012).

[22] M. Venuti, M. Stener, and P. Decleva, Chem. Phys. 234, 95 (1998).

[23] D. Toffoli, M. Stener, G. Fronzoni, and P. Decleva, Chem. Phys. 276, 25 (2012).

[24] U. Hergenhahn, J. Phys. B 37, R89 (2004).

[25] K. P. Huber and G. Herzberg, in NIST Chemistry WebBook, NIST Standard Reference Database Number 69, edited by P. J. Linstrom and W. G. Mallard (National Institute of Standards and Technology, Gaithersburg, MD), http://webbook.nist.gov (retrieved February 14, 2013).

[26] J. B. Williams, J. Phys. B 45, 194003 (2012).

[27] T. Hartman, P. N. Juranić, K. Collins, B. Reilly, N. Appathurai, and R. Wehlitz, Phys. Rev. Lett. 108, 023001 (2012).

[28] P. Sałek, F. Gel'mukhanov, H. Ågren, O. Björneholm, and S. Svensson, Phys. Rev. A 60, 2786 (1999).

[29] F. Gel'mukhanov, P. Sałek, and H. Ågren, Phys. Rev. A 64, 012504 (2001)

[30] H. M. Köppe, A. L. D. Kilcoyne, J. Feldhaus, and A. M. Bradshaw, J. Electron Spectrosc. Relat. Phenom. 75, 97 (1995).

[31] M. Matsumoto, K. Ueda, E. Kukk, H. Yoshida, T. Tanaka, M. Kitajima, H. Tanaka, Y. Tamenori, K. Kuramoto, M. Ehara, and H. Nakatsuji, Chem. Phys. Lett. 417, 89 (2006).

[32] E. Shigemasa, J. Adachi, K. Soejima, N. Watanabe, A. Yagishita, and N. A. Cherepkov, Phys. Rev. Lett. 80, 1622 (1998). 